\title{
Geometric Summary of the 9 Chip Ladder for the Do Silicon Tracker
}

\author{
P. Ratzmann, W. Cooper, D. Goloskie, \\ J. Kowalski, R. Lipton, P. Rapidis, C. Serritella
}

D0EN-3823.112-EN-468

November 18, 1997

\section{Geometry}

Two hybrids types are required to accomodate the flipping of ladders within each bulkhead layer, in order to account for the pigtail routing. Left and right versions are shown below, following the definitions laid out by Mike Matulik. These drawings are not to proper scale in the sketches below. The dimensionally correct versions of the 9 chip hybrids are stored in DCS under drawing number 3823.112-MD-317803 for the lefthanded version, and 3823.112-MD-317804 for the right handed version.

Handedness of the hybrids are designated as shown in the figures and table below. There are long and short versions of both the left and the right, for four total 9 chip hdi designs. The pigtail lengths of the long and short are shown in a table in the hybrid drawings which reside in DCS.

The chamfer in the hybrid corners ( $\mathrm{N}$ side) is placed in order to enable the hybrid to be glued to the beryllium substrate, whereas the rectangular cuttout on the same side is to allow direct gluing of a temperature sensor to the substrate metal. The oblong shape on the $\mathrm{N}$ side of both hybrids is a "stay-clear" region (defined in the final drawings) where pressure will be applied to the hybrid during the second stage of ladder construction.

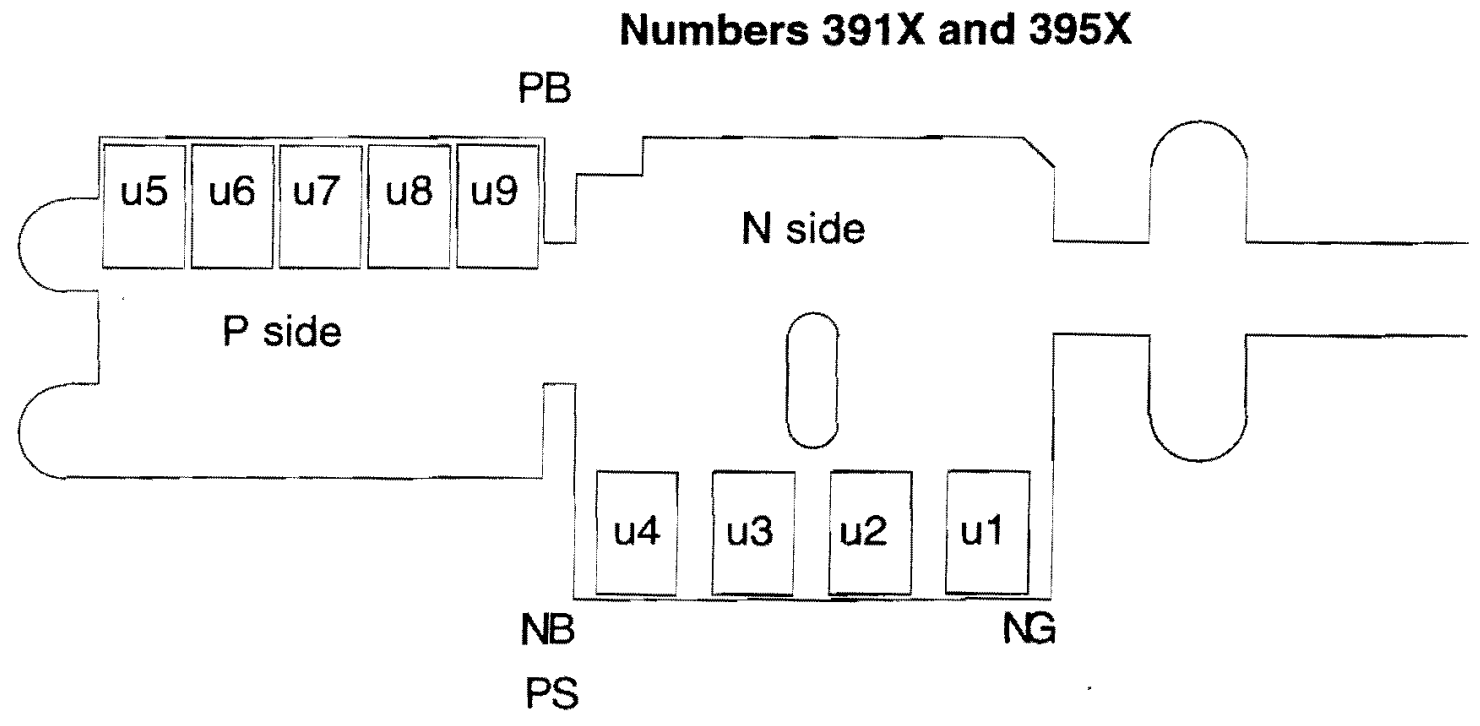

Left-handed 9 chip hybrid 


\section{Numbers $392 X$ and $396 X$}

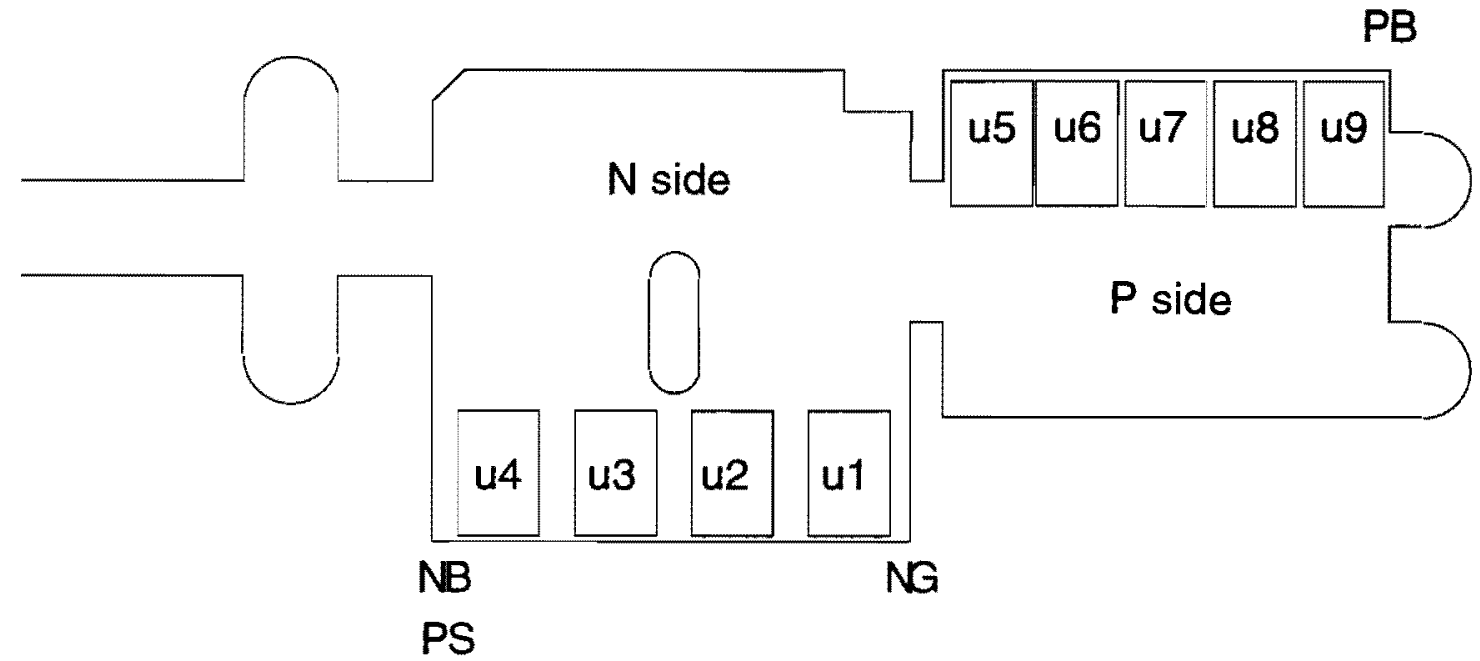

Right-handed 9 chip hybrid

There are two types of 9 chip silicon detectors as well; $-2^{\circ}$ (defined as an A type) and $+2^{\circ}$ (B type) on the stereo side. These masks are not identical, they have the P-stop bonding location on the same side of the detector for both, as shown in the sketches below.

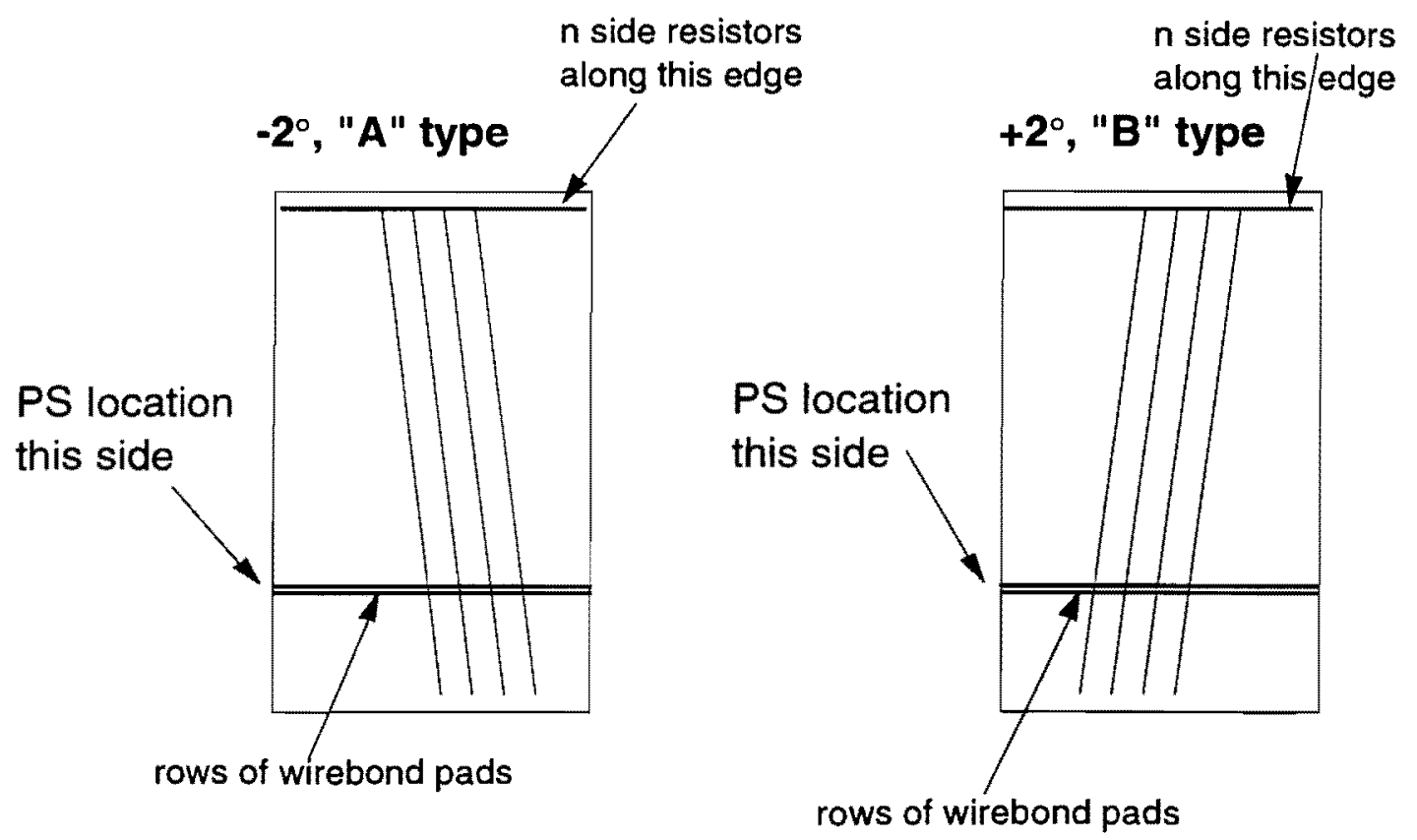

Detector types

There are four ladder designs which accomodate the pigtail orientation required and the proper $+/-2^{\circ}$ orientation of strips in the barrel. Mounted in the barrels as such results in all detectors of L2 with the same strip orientation in the global detector coordinate system, as well as a switch in the strip orientation of the global system in L4. 


\section{Type 2}

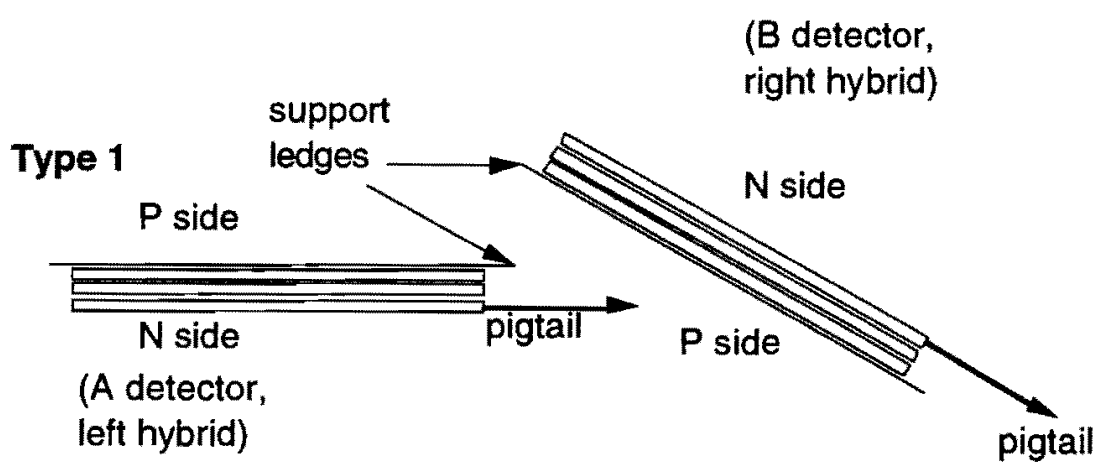

Two ladders mounted in the bulkhead-L2

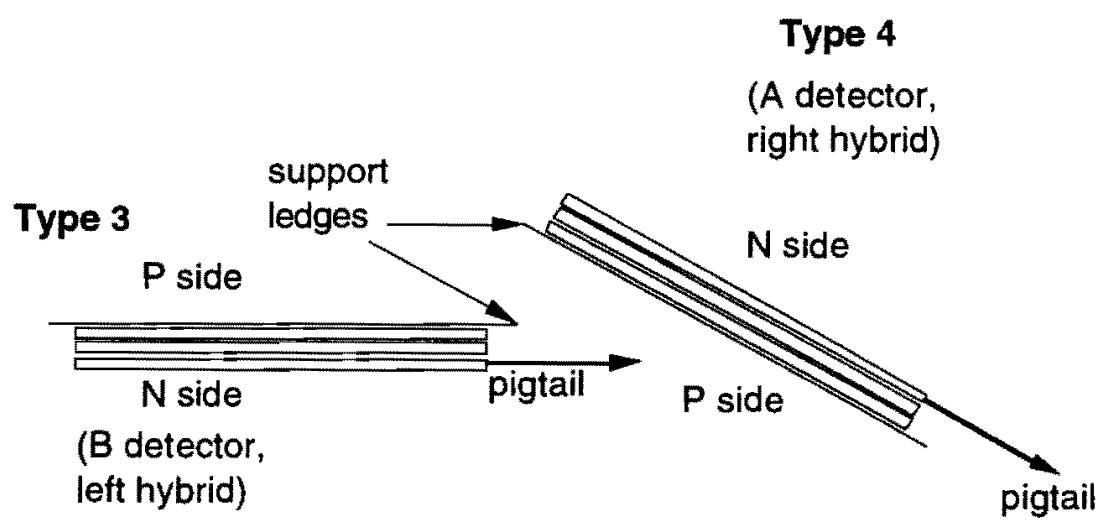

Two ladders mounted in the bulkhead-L4

The cable length is determined by the cable path and the radial location in the barrel. A short and long version of $\mathrm{R}$ and $\mathrm{L}$ hybrids requires a differing amount of $\mathrm{R}$-short, $\mathrm{R}$-long, L-short, and L-long hybrids as seen in the table. The identifier numbers shown in the table were selected by Mike Matulik and are described in a note written by him entitled "D0 Silicon Vertex Detector High Density Interconnect Circuit Identification". 3951, for instance, is the first "left-long" hybrid with subsequent iterations to the design following in numerical order.

\begin{tabular}{|c|c|l|l|l|l|}
\hline layer & ladder type & $\begin{array}{l}\text { detector } \\
\text { type }\end{array}$ & $\begin{array}{l}\text { hybrid } \\
\text { handedness }\end{array}$ & $\begin{array}{l}\text { number } \\
\text { per barrel }\end{array}$ & identifier \\
\hline 2 & 1 & A & left-long & 6 & $3951-59$ \\
\hline 2 & 2 & B & right-long & 6 & $3961-69$ \\
\hline 4 & 3 & B & left-short & 12 & $3911-19$ \\
\hline 4 & 4 & A & right-short & 12 & $3921-29$ \\
\hline
\end{tabular}

Table of 9 chip ladder types and counts, per barrel 


\section{Wirebonding}

Wirebonding of the P-stops is dictated by the access locations to the P-stops as shown in the detector sketches above. Bias and guard ring wirebonding can be done on either edge of the detectors. The locations shown for these bonds was selected based on the plan view sketches by Mike Matulik.

\section{Fixturing and assembly sequence}

Fixturing is designed for the following sequence of steps:

1. Align silicon

2. Place P side (phi strips) hybrid on top of silicon with adhesive, between posts, and allow to cure.

3. Wirebond SVX II/silicon, silicon/silicon, P side bias and guard ring from hybrid/silicon and silicon/silicon.

4. Glue on rails and allow to cure.

5. Flip assembly.

6. Wrap and glue $\mathrm{N}$ side (stereo) hybrid to silicon surface.

7. Wirebond SVX II/silicon, silicon/silicon, $\mathrm{N}$ side bias, guard ring, and P-stop from hybrid/silicon and silicon/sillicon. 